\title{
Subchondral bone remodeling: comparing nanofracture with microfracture. An ovine in vivo study
}

\author{
PIETRO ZEDDE ${ }^{1}$, SEBASTIANO CUDONI ${ }^{1}$, GIACOMO GIACHETTI ${ }^{2}$, MARIA LUCIA MANUNTA ${ }^{3}$, \\ GEROLAMO MASALA ${ }^{3}$, ANTONIO BRUNETTI ${ }^{4}$, ANDREA FABIO MANUNTA $^{2}$
}

${ }^{1}$ Orthopaedic and Traumatology Unit, Hospital San Francesco, Nuoro, Italy

${ }^{2}$ Orthopaedic Department, University of Sassari, Italy

${ }^{3}$ Department of Veterinary Medicine, University of Sassari, Italy

${ }^{4}$ Polcoming Department, University of Sassari, Italy

\begin{abstract}
Purpose: microfracture, providing direct stimulation of chondrogenic mesenchymal stem cells (MSCs) in the subchondral bone, remains the most frequently used primary cartilage repair technique. However, the newly formed type I collagen-rich fibrocartilaginous tissue has poor biomechanical properties and a tendency to degenerate. To overcome these limitations the nanofracture technique was introduced. Our purpose was to compare subchondral bone remodeling 6 months after microfracture versus nanofracture (subchondral needling) treatment in an ovine model.

Methods: full-thickness chondral lesions were created in the load-bearing area of the medial femoral condyles in four adult sheep. Each animal was then treated on one side with microfracture and on the contralateral side with nanofracture. Subchondral bone remodeling was assessed by micro-CT using a Bruker $^{\circledR}$ SKYSCAN and CTVOX 2.7 software (Bruker Corp., Billerica, MA, USA) for image reconstruction; trabecular bone density measurements were performed through a color-representation structure thickness analysis.
\end{abstract}

Results: at the six-month endpoint, the microfracturetreated samples showed limited perforation depth and cone-shaped channels with large diameters at the joint surface. The channel walls displayed a high degree of

\section{Corresponding Author:}

Pietro Zedde, MD

Orthopaedic and Traumatology Unit

Hospital San Francesco

Via Mannironi 1, 08100 Nuoro, Italy

E-mail: pietrozedde@tiscali.it regularity with significant trabecular bone compaction leading to a sealing effect with limited communication with the surrounding trabecular canals. Condyles treated with nanofracture showed channels characterized by greater depth and smaller diameters and natural irregularities of the channel walls, absence of trabecular compaction around the perforation, remarkable communication with trabecular canals, and neo-trabecular remodeling inside the channels.

Conclusions: nanofracture is an effective and innovative repair technique allowing deeper perforation into subchondral bone with less trabecular fragmentation and compaction when compared to microfracture; it results in better restoration of the normal subchondral bone architecture at six months.

Clinical Relevance: our data support the use of smaller-diameter and deeper subchondral bone perforation for MSC stimulation; this technique may prove to be an attractive alternative to standard microfracture procedures.

Keywords: microfracture, nanofracture, subchondral bone, mesenchymal stem cell stimulation, ovine, micro-CT.

\section{Introduction}

Many treatments and new biomaterials for cartilage repair have been introduced over the past 30 years, but the microfracture technique developed by Steadman et al. (1) remains the most frequently used. The biological rationale for this repair technique is that direct stimulation of mesenchymal stem cells (MSCs) in the subchondral bone leads these cells to express their 
chondrogenic potential (2-6). The advantages of this technique include its minimal invasiveness, favorable cost-effectiveness ratio, and low technical demands. It is giving positive results, especially in young patients with defects of limited size $(4,7,8)$. The quality and characteristics of the newly formed fibrocartilaginous tissue, however, are much debated in the literature (911), due to the limited biomechanical properties of this type I collagen-rich tissue and its tendency to degenerate.

In recent years, improved knowledge and understanding of the structural and functional architecture of subchondral bone have led to an increased focus on the need to preserve its integrity during chondral defect treatment. The limited penetration depth of the microfracture awl into subchondral bone and the resulting trabecular compaction around the perforations are currently thought to be among the causes of the fibrocartilaginous tissue formation associated with this technique.

In order to overcome the mechanical limitations of the microfracture method, the nanofracture technique (Arthrosurface, Franklin, MA, USA), using smallerdiameter and deeper subchondral bone needle perforations (5), was recently introduced. The purpose of the present study was to compare subchondral bone remodeling after treatment of full-thickness chondral defects with microfracture versus nanofracture in an ovine knee model. Our hypothesis was that nanofracture-treated defects would show less subchondral bone compaction around the channels and better preservation of the trabecular structure compared with microfracture-treated ones.

\section{Methods}

This study was approved by the Ethics Committee of the University of Sassari, Italy and all procedures were conducted in accordance with the institutional animal care regulations, which comply with the National Institute of Health Guidelines on the Care of Laboratory Animals.

\section{Surgical technique}

Four adult Sardinian ewes, aged 5.5 years and weighing approximately $45 \mathrm{~kg}$ were used in the study. All the animals were examined and found to be in good health.
Surgery was performed in sterile conditions and with the sheep under general anesthesia. All the sheep were intubated after administration of thiopentone (25 $\mathrm{mg} / \mathrm{kg}$ ) and ventilated with $\mathrm{O}_{2}$ in $\mathrm{N}_{2} \mathrm{O}$ by volume control. Anesthesia was maintained with 1.5 to $2 \%$ isoflurane; a bolus dose of $0.1 \mathrm{mg}$ of fentanyl was given before surgery.

In all the animals, a medial parapatellar arthrotomy was performed on the right and left stifle. The incision was performed in such a way as to expose the medial femoral condyle in both hind legs. An 8-mm diameter (area: $50.3 \mathrm{~mm}^{2}$ ), full-thickness chondral lesion in the load-bearing area of each medial femoral condyle was created using an arthroscopic burr (Fig. 1). The calcified layer was removed; vertical walls were created at the periphery of the cartilage lesion. The defects were then treated using microfracture on one side and nanofracture on the contralateral side (Fig. 2). Each cartilage lesion was treated with three or five channels. In accordance with published recommendations, the distance between each channel was $3 \mathrm{~mm}$ (3).

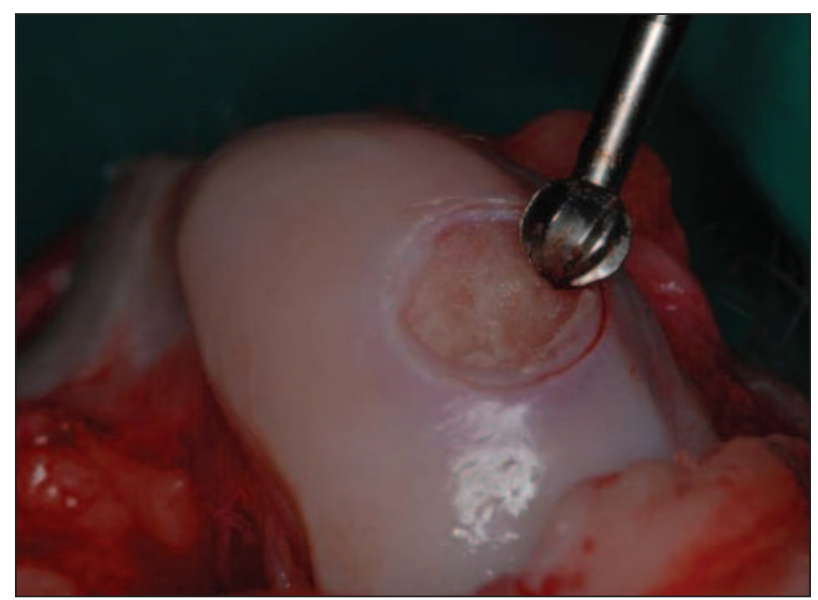

Fig. 1. A full-thickness chondral lesion in the load-bearing area of each medial femoral condyle was created using an arthroscopic burr.

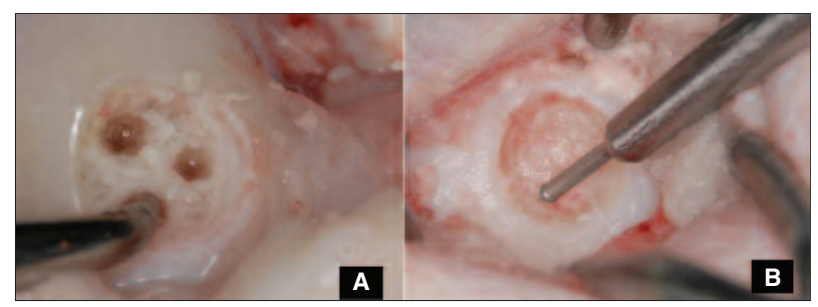

Fig. 2. A: Treatment of the defect with microfracture. B: Treatment of the defect with nanofracture. 
Microfracture sites were treated using a curved Steadman awl. The perforation depth was user controlled with visual feedback from the awl tip. Nanofracture sites were treated using a cannulated awl and a 1-mm thick Nitinol needle (Arthrosurface, Franklin, MA, USA). The 9-mm perforation depth of the needle is controlled by the awl. The surgical technique is described in the literature (5).

Upon completion of the cartilage repair procedures, all incisions were closed in layers according to standard surgical practice. Postoperatively, the animals were kept in stalls with limited movement and weight bearing. After surgery, the animals were left free to roam in their fencings without any immobilization of the operated limb. Full weight bearing was allowed as tolerated and no specific exercise regimen was adopted. General health and weight-bearing status were monitored by a vet during recovery.

\section{Micro-CT}

Six months after surgery, the sheep were euthanized according to the ethics committee approved protocol. All treated joints were harvested, stripped of soft tissues and a $2 \times 2 \mathrm{~cm}$ condylar specimen that contained the treated lesion was prepared for micro-CT imaging. Subchondral bone remodeling was assessed by microCT using a Bruker ${ }^{\circledR}$ SKYSCAN and CTVOX 2.7 software (Bruker Corp., Billerica, MA, USA) for image reconstruction.

The SKYSCAN inspection machine allows high-resolution, 3D scanning of small objects, and provides morphometric information about the analyzed sample. The device has an X-ray generator, a rotating sample holder and a charge coupled device (CCD) camera. The equipment, controlled through a dual processor computer and dedicated software (CTVOX 2.7), generates 3D images and calculates morphometric parameters. The SKYSCAN X-ray microtomography system is based on a microfocus X-ray tube and an 11Mpixel CCD camera with a pixel size of 9x9 microns square. The X-ray tube operates at up to $100 \mathrm{kV}$ and $200 \mu \mathrm{A}$; it emits brems strahlung cone beam radiation. Brems strahlung beams are produced by directing energyanalyzed electrons onto a tantalum conversion target at the end of the horizontal beam line. The tomographic images are reconstructed as described by Feldkamp et al. using a convolution back-projection method (12).
The reconstruction algorithm runs on a graphics processing unit-based system that makes the reconstruction task quicker. The maximum permitted sample size depends on the magnification, but must be under $5 \mathrm{~cm}$. The maximum spatial resolution is about $0.5 \mu \mathrm{m}$ for a sample with a diameter of 1-2 $\mathrm{mm}$. In the measurements reported here the following $\mathrm{X}$-ray tube setup was used: $77 \mathrm{kV}, 127 \mu \mathrm{A}$ with a $0.5-\mu \mathrm{m}$ thick $\mathrm{Al}$ filter. Projections were taken at 0.2 degree intervals over $360^{\circ}$ for a total of 1800 projections. Each projection required $1.3 \mathrm{~s}$ and was formed by $4000 \times 4000$ pixels. The spatial resolution was about $4.9 \mu \mathrm{m}$. About 2300 slices were reconstructed in 21 hours and were visualized in 3D using the CTVOX software by Bruker ${ }^{\circledR}$. In order to improve the contrast a false color representation system was also used. The color red represented reduced density in the bone, cyan standard density, and green intermediate density between reduced and standard. Of course, this system can be used only for the purpose of obtaining better visual representation, as the Hounsfield scale is the standard scale to use.

\section{Image analysis}

The tomographic image was reconstructed by measuring the X-ray beam attenuation in several parallel trajectories through the condyles, assessing the attenuation component of single voxels based on a specific reconstruction algorithm, and finally displaying the reconstructed numerical values. The depth of each channel was measured; the structure of the surrounding tissue was analyzed; communication between preexisting trabecular canals and the perforation was also compared. Trabecular bone density measurements were performed through a color-representation structure thickness analysis and compared between the two groups.

\section{Results}

Micro-CT of the microfracture samples showed a limited perforation depth of the awl. The cone-shaped channels had large diameters at the joint surface; the channel walls displayed a high degree of regularity with significant trabecular bone compaction, leading to a sealing effect and therefore limited communication of the channels with the surrounding trabecular 


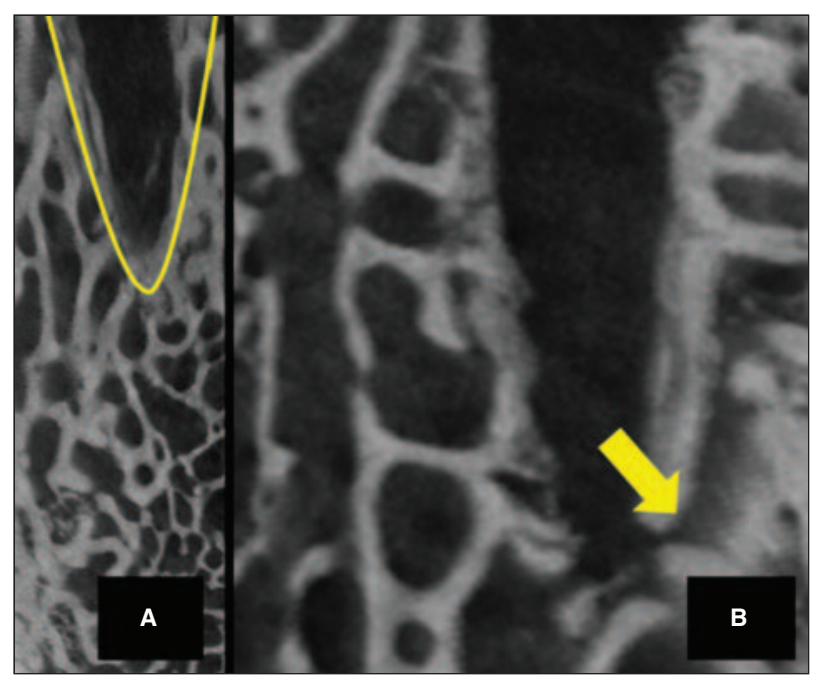

Fig. 3. A: The cone-shaped channels had large diameters at the joint surface with significant trabecular bone compaction. B: Limited communication of the channel with the surrounding trabecular canalsafter microfracture.

canals (Fig. 3), and the development of newly formed trabeculae inside the channels. In three of the four microfracture samples, subchondral cysts ranging from $7 \mathrm{~mm}$ to $12 \mathrm{~mm}$ in diameter were found near the perforation (Fig. 4).

Micro-CT scans of the nanofracture-treated condyles showed a greater depth of the perforations, which had a smaller diameter compared to the microfracture ones, with natural irregularities of the channel walls, absence of trabecular compaction around the perforations, and remarkable communication between preexisting canals and the perforation (Fig. 5), as well as neo-trabecular remodeling inside the channels. None of the nanofracture samples showed subchondral cyst formation.

Color-representation structure analysis in the nanofracture-treated condyles showed less trabecular fragmentation surrounding the channels as well as intrachannel bone remodeling, resulting in a trabecular structure remarkably similar to the native subchondral bone (Fig. 6).

\section{Discussion}

The key finding of this study is that small-diameter needle perforation into full-thickness condylar defects showed less subchondral bone compaction and better

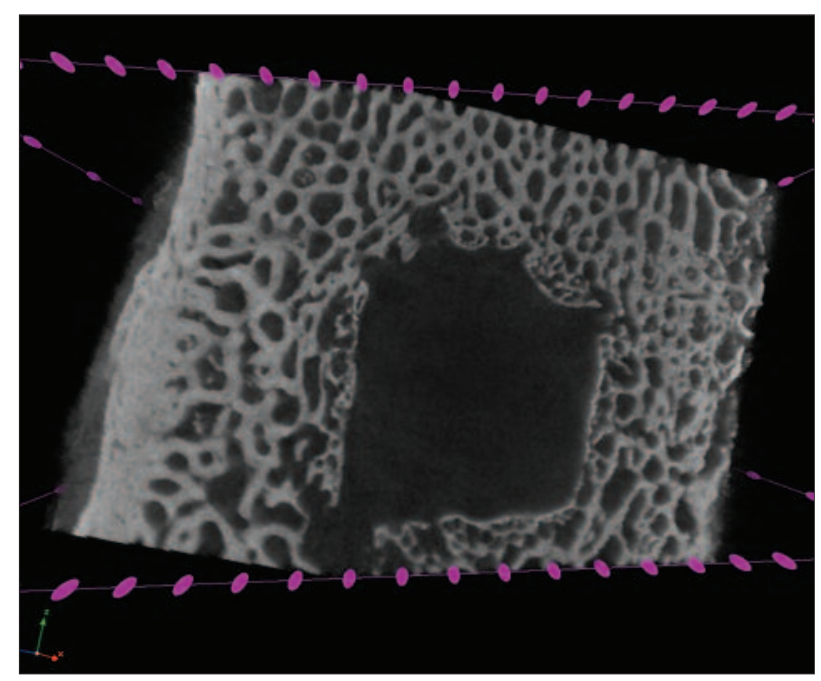

Fig. 4. Subchondral cysts ranging from $7 \mathrm{~mm}$ to $12 \mathrm{~mm}$ were found near the perforation after microfracture.

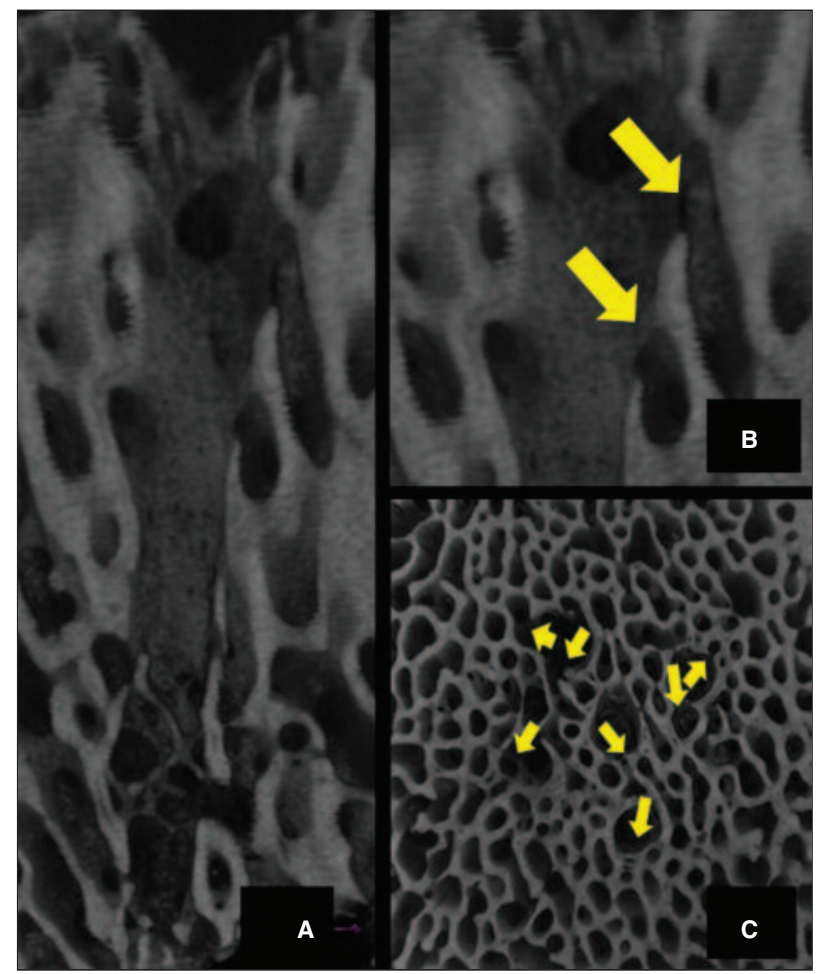

Fig. 5. A: Micro-CT scan of the condyles treated with nanofracture showing perforations with a greater depth and a smaller diameter compared to the perforations in the microfracture-treated ones, natural irregularities of the channel walls, and absence of trabecular compaction around the perforations. B: Remarkable communication between pre-existing trabecular canals and the perforation after between pre-existing trabecular canals and the perforation after
nanofracture - Sagittal view. C: Remarkable communication between pre-existing trabecular canals and the perforation after nanofracture - Axial view. 


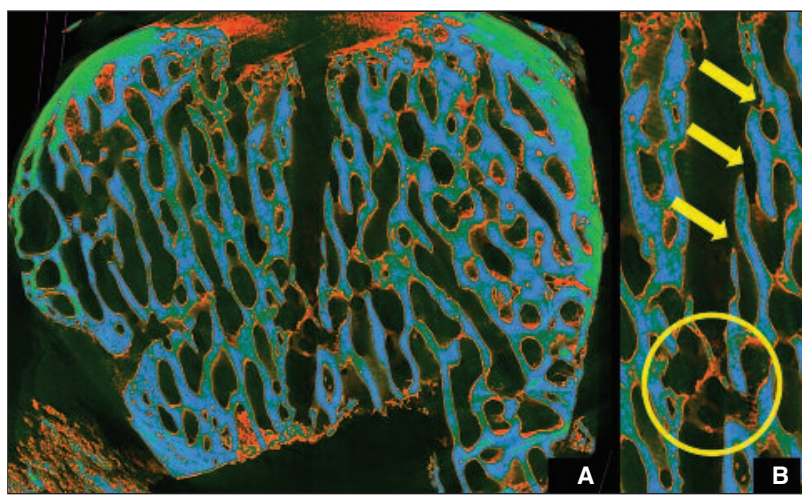

Fig. 6. A: Color-representation structure analysis in the nanofracturetreated condyles. B: Less trabecular fragmentation around the channels as well as intra-channel bone remodeling with a trabecular structure remarkably similar to that of native subchondral bone.

preservation of trabecular structures when compared with microfracture treatment. A secondary finding is that the bone remodeling after nanofracture resulted in a trabecular structure remarkably similar to that of native subchondral bone.

Since the first use of the microfracture technique, by Steadman in the 1980s, it has become the treatment of choice for the management of chondral lesions (1). The technique is based on direct stimulation of MSCs of the subchondral bone which have a high potential for differentiation into various connective tissues, including cartilage, bone, tendon and ligaments $(13,14)$. MSCs achieve their regenerative effects by promoting tissue repair and the resolution of inflammation, both through direct cell-to-cell interaction and through the secretion of bioactive components $(15,16)$.

Over the years, several fundamental aspects of the microfracture technique have become better documented and better understood. Several studies, reporting clinical improvement from baseline conditions in $70-90 \%$ of patients, have demonstrated its validity (3, $4,8,17-19)$. However the formation of fibrocartilaginous tissue and the deterioration of the clinical results over time are major limitations (20-23), and alternative procedures for the management of chondral lesions have been suggested $(9,10)$.

Chen et al. published several studies evaluating the histological characteristics of the newly formed tissue after treatment of chondral defects with different repair techniques showing various degrees of penetration into the subchondral bone (24-26). Histological and histomorphometric findings showed that the best access to bone marrow was obtained when drilling to greater depths, as this led to improved defect filling and the production of cartilage with a higher hyaline content (25). In another study, the same group showed that treatment with microfracture is associated with fragmentation and compaction around the trabecular canals (24). Examining the results of microfracture and drilling at three months, they found incomplete filling, cyst formation, bone overgrowth and poor bone integration with both techniques (26).

Orth et al. (27) recently published the results of a comparative study in which $8 \times 4 \mathrm{~mm}$ full-thickness defects in the weight-bearing area of ovine condyles were treated using two custom awls with two different diameters: $1 \mathrm{~mm}$ and $1.2 \mathrm{~mm}$. The perforation depth was stop controlled at $5 \mathrm{~mm}$; the distal tips were trihedral. The Authors reported a significant improvement in the overall histological score using small-diameter awls. In addition, the histological surface regularity was significantly improved with the smaller instruments (27).

Our study, showing beneficial subchondral effects when using small-diameter needle perforation, is in agreement with these findings. While Orth et al. used custom instruments and chose a $5-\mathrm{mm}$ perforation depth, we used clinically available instrumentation for nanofracture, in which a 1-mm thick needle is inserted into a cannulated awl that creates stop-controlled perforations with a depth of $9 \mathrm{~mm}$. This technique was compared with the microfracture technique, performed using awls with the standard cone-shaped tip. Our study confirmed previous micro-CT findings. Microfracture demonstrated limited perforation depth and trabecular bone compression leading to an apparent sealing effect inside the channel, and thus a change from its native physiological appearance $(24,28)$. Our results are also consistent with previous reports of subchondral cyst formation following microfracture $(26,29,30)$. Orth et al. (29) reported cyst formation and osteochondral osteophytes in $74 \%$ of their ovine condyles treated with subchondral drilling. They also noted that the original microarchitecture was not reestablished. Hoeman et al. (30) suggested that excessive drilling or communicating holes combined with early weight bearing could lead to subchondral cyst formation.

Our 3D reconstruction of the ovine condyles after nanofracture showed lower exposure of the osteo- 
chondral surface, less fragmentation and compaction inside the channels, and therefore better communication with pre-existing trabecular canals. These canals allow the immediate on-site formation of a super-clot (Fig. 7), which results in improved recruitment of multi-potent MSCs.

Nanofracture also led to the formation of a neo-trabecular arrangement inside the channel which was similar to the native subchondral bone architecture and did not show any signs of subchondral cyst formation. Furthermore, the channel depth was enhanced, the channel diameter was reduced, and the trabecular bone architecture of the channel wall largely maintained its physiological appearance.

These comparative observations can be explained on the basis of the mechanical principles by which the subchondral channels are created. Both the procedures examined in this study are based on the application of axial force without rotation, however, the cone-shaped tip of the wider microfracture awl leads to bone displacement through compaction.

Conversely, the thin nanofracture needle tip creates smaller trabecular fractures with less particle fragmentation and no apparent bone compaction, resulting in a large number of open canals that communicate with the nanofracture channel.

Overall, the inferences that can be drawn from animal studies like the present one are limited because functional status cannot be assessed at baseline and followup evaluations. However, the animal model used in this study is considered suitable for cartilage defect

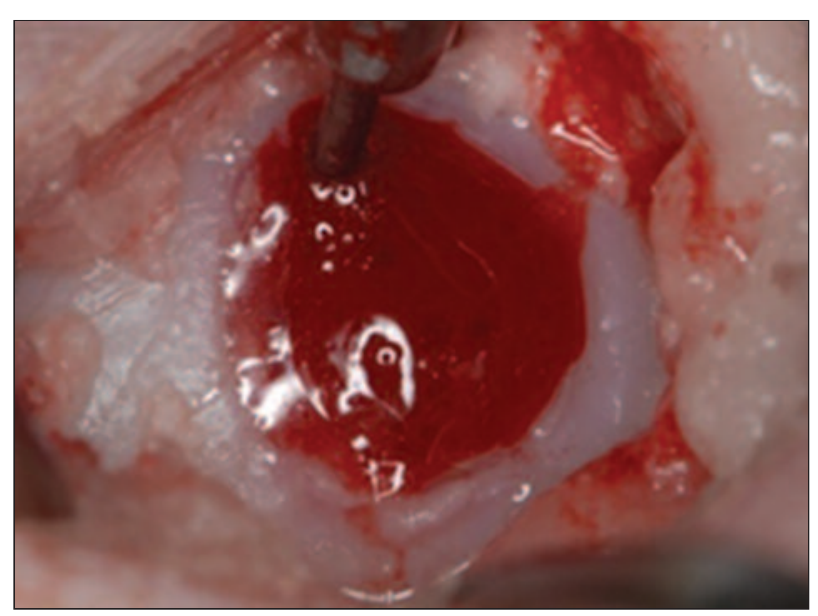

Fig. 7. Immediate on-site formation of a super-clot after nanofracture. testing. A further limitation of this study is the small cohort size. Its strength, on the other hand, is that it was performed using clinically available instrumentation, applied in accordance with previously described methods (5).

In conclusion, our hypothesis was proven correct as nanofracture was found to be an effective and innovative repair technique that allows deeper perforation into the subchondral bone while resulting in less trabecular fragmentation and compaction when compared with microfracture. The resulting communication with a large number of native trabecular canals allows better bone marrow access and therefore greater pluripotent MSC recruitment for the restoration of normal subchondral bone architecture.

In order to better understand the results of nanofracture treatment of chondral defects, well-designed investigations in human subjects are required. These should include adequate histological and biomechanical evaluations of the newly formed tissue and longterm clinical and functional outcomes.

\section{References}

1. Steadman JR, Rodkey WG, Rodrigo JJ. Microfracture: surgical technique and rehabilitation to treat chondral defects. Clin Orthop Relat Res. 2001;(391 Suppl): S362-S369.

2. Khan WS, Johnson DS, Hardingham TE. The potential of stem cells in the treatment of knee cartilage defects. Knee. 2010;17:369-374.

3. Mithoefer K, Williams RJ 3rd, Warren RF, et al. The microfracture technique for the treatment of articular cartilage lesions in the knee. A prospective cohort study. J Bone Joint Surg Am. 2005;87:1911-1920.

4. Mithoefer K, Williams RJ 3rd, Warren RF, et al. Chondral resurfacing of articular cartilage defects in the knee with the microfracture technique. J Bone Joint Surg Am. 2006;88 Suppl 1 pt 2:294-304.

5. Benthien JP, Behrens P. Reviewing subchondral cartilage surgery: considerations for standardised and outcome predictable cartilage remodeling: a technical note. Int Orthop 2013; 37:2139-2145.

6. Shapiro F, Koide S, Glimcher MJ. Cell origin and differentiation in the repair of full-thickness defects of articular cartilage. J Bone Joint Surg Am. 1993;75:532-553.

7. Kreuz PC, Steinwachs MR, Erggelet C, et al. Results after microfracture of full-thickness chondral defects in different compartments in the knee. Osteoarthritis Cartilage. 2006; 14: 1119-1125.

8. Mithoefer K, Williams RJ 3rd, Warren RF, et al. High-impact athletics after knee articular cartilage repair: a prospective evaluation of the microfracture technique. Am J Sport. 2006; 34:1413-1418.

9. Saris DB, Vanlauwe J, Victor J, et al. Characterized chondrocyte implantation results in better structural repair when 
treating symptomatic cartilage defects of the knee in a randomized controlled trial versus microfracture. Am J Sports Med. 2008;36:235-246.

10. Saris DB, Vanlauwe J, Victor J, et al. Treatment of symptomatic cartilage defects of the knee: characterized chondrocyte implantation results in better clinical outcome at 36 months in a randomized trial compared to microfracture. Am J Sports Med. 2009;37 Suppl 1:10S-19S.

11. Vanlauwe J, Saris DB, Victor J, et al. Five-year outcome of characterized chondrocyte implantation versus microfracture for symptomatic cartilage defects of the knee: early treatment matters. Am J Sports Med. 2011;39:2566-2574.

12. Feldkamp LA, Davis LC, Kress JW. Practical cone-beam algorithm. J Opt Soc Am A. 1984;1:612-619.

13. Caplan AI, Dennis JE. Mesenchymal stem cells as trophic mediators. J Cell Biochem. 2006;98:1076-1084.

14. Lodi D, Iannitti T, Palmieri B. Stem cells in clinical practice: applications and warnings. J Exp Clin Cancer Res. 2011;30:9.

15. Caplan AI. Review: mesenchymal stem cells: cell-based reconstructive therapy in orthopedics. Tissue Eng. 2005;11: 1198-1211.

16. Chen FH, Tuan RS. Mesenchymal stem cells in arthritic diseases. Arthr Res Ther. 2008;10:223.

17. Steadman JR, Rodkey WG, Singleton SB, et al. Microfracture technique for full-thickness chondral defects. Technique and clinical results. Oper Tech Orthop. 1997;7:300-304.

18. Steadman JR, Miller BS, Karas SG, et al. The microfracture technique in the treatment of full-thickness chondral lesions of the knee in National Football League players. J Knee Surg. 2003;16:83-86.

19. Steadman JR, Briggs KK, Rodrigo J, et al. Outcomes of microfracture for traumatic chondral defects of the knee: average 11-year follow-up. Arthroscopy. 2003;19:477-484.

20. Kon E, Gobbi A, Filardo G, et al. Arthroscopic second-generation autologous chondrocyte implantation compared with microfracture for chondral lesions of the knee; prospective nonrandomized study at 5 years. Am J Sports Med. 2009; $37: 33-42$.
21. Kon E, Filardo G, Berruto M, et al. Articular cartilage treatment in high-level male soccer players: a prospective comparative study of arthroscopic second-generation autologous chondrocyte implantation versus microfracture. Am J Sport Med. 2011;39:2549-2557.

22. Mithoefer K, McAdams T, Williams RJ, et al. Clinical efficacy of the microfracture technique for articular cartilage repair in the knee: an evidence-based systematic analysis. Am J Sports Med. 2009;37:2053-2063.

23. Namdari S, Baldwin K, Anakwenze O, et al. Results and performance after microfracture in National Basketball Association athletes. Am J Sports Med. 2009;37:943-948.

24. Chen H, Sun J, Hoeman CD, et al. Drilling and microfracture lead to different bone structure and necrosis during bonemarrow stimulation for cartilage repair. J Orthop Res. 2009;27:1432-1438.

25. Chen H, Hoemann CD, Sun J, et al. Depth of subchondral perforation influences the outcome of bone marrow stimulation cartilage repair. J Orthop Res. 2011;29:1178-1184.

26. Chen H, Chevrier A, Hoemann CD, et al. Characterization of subchondral bone repair for marrow-stimulated chondral defects and its relationship to articular cartilage resurfacing. Am J Sports Med. 2011;39:1731-40.

27. Orth P, Duffner J, Zurakowski D, et al. Small-diameter awls improve articular cartilage repair after microfracture treatment in a translational animal model. Am J Sports Med. 2016;44:209-219.

28. Fortier LA, Cole BJ, Mcllwraith CW. Science and animal models of marrow stimulation for cartilage repair. J Knee Surg. 2012;25:3-8.

29. Orth P, Goebel L, Wolfram U, et al. Effect of subchondral drilling on the microarchitecture of subchondral bone: analysis in a large animal model at 6 months. Am J Sports Med. 2012;40:828-836.

30. Hoemann CD, Hurtig M, Rossomacha E, et al. Chitosangylcerolphosphate/blood implants improve hyaline cartilage repair in ovine microfracture defects. J Bone Joint Surg Am. 2005;87:2671-2686. 